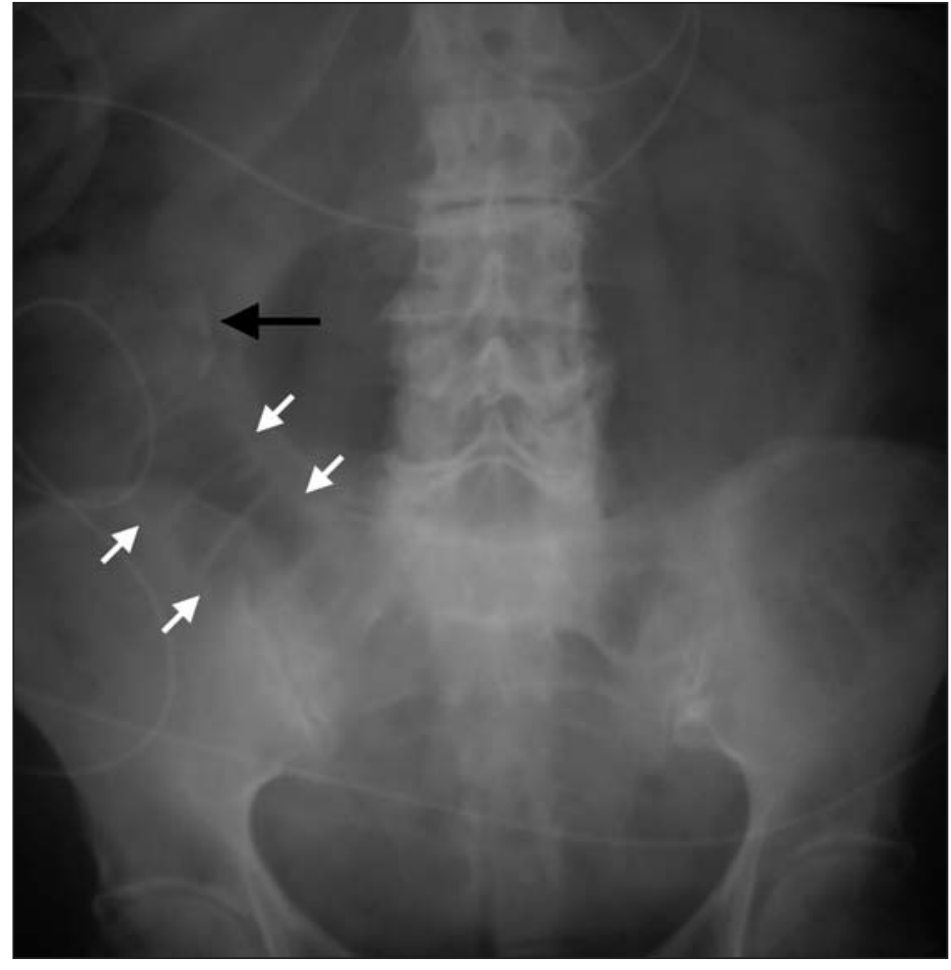

Figure 1: Radiograph of the abdomen of an elderly woman who was admitted with signs and symptoms suggestive of obstructive ileus. The film shows a gallstone within the bowel (black arrow) and dilated small-bowel loops (white arrows) consistent with obstruction.
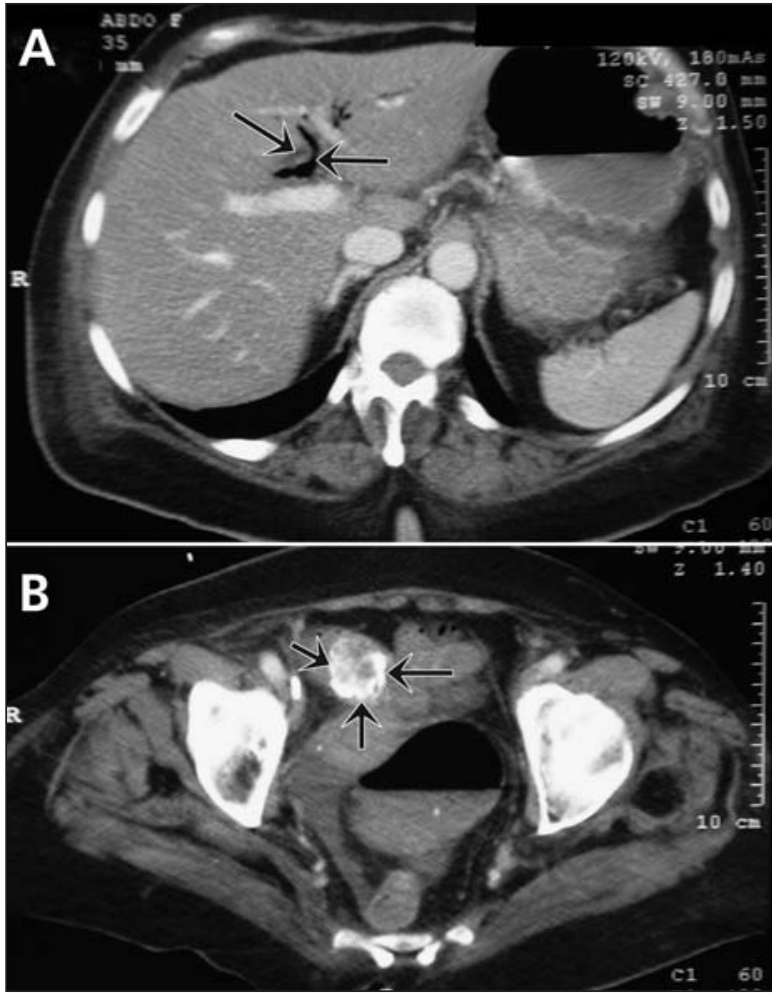

Figure 2: Multidetector computed tomography scans, showing air in the biliary tree ( $A$, arrows) and an ectopic gallstone in the intestinal lumen ( $B$, arrows).

\title{
Gallstone ileus
}

$\Lambda$ n elderly woman in her 90s was admitted with signs and symptoms suggestive of obstructive ileus: crampy abdominal pain, repeated episodes of vomiting and increased bowel sounds with tympanic sound on percussion. A plain film radiograph (Figure 1) and computed tomography scans (Figure 2) of her abdomen confirmed Rigler's triad: ectopic gallstone, pneumobilia and small-bowel dilation. This patient's gallstone $(2.5 \times 3.5 \mathrm{~cm})$ was lodged in the jejunum. Because the patient had concurrent disorders and was a poor operative candidate, we chose a conservative approach to treatment. Unfortunately, the patient died.

Gallstone ileus is a rare complication of gallstone disease. A small-bowel obstruction results after 1 or more gallstones migrate through a cholecystoenteric fistula. ${ }^{1}$ Elderly women are more likely than men to experience this complication for unclear reasons. Among patients with comorbidities, gallstone ileus has a mortality rate of up to $18 \%{ }^{2}$ In patients at low operative risk, staged laparoscopic management of gallstone ileus and of the associated cholecystoenteric fistula gives good results. For the majority of patients, though, laparotomy is the treatment of choice.
Anastasios Koulaouzidis MD Department of Gastroenterology Warrington Hospital

Warrington, UK John Moschos MD PhD

Department of Gastroenterology General Army Hospital

Thessaloniki, Greece Shivaram Bhat MB BCh

Department of Internal Medicine Warrington Hospital

Karthikeyan Gopal MBBS

Department of Radiology

Warrington Hospital

Warrington, UK

\section{REFERENCES}

1. Abou-Saif A, Al-Kawas FH. Complications of gallstone disease: Mirizzi syndrome, cholecystocholedochal fistula and gallstone ileus. Am J Gastroenterol 2002;97:249-54.

2. Moschos J, Pilpilidis I, Antonopoulos $\mathrm{Z}$, et al. Complicated endoscopic management of Bouveret's syndrome. A case report and review. Rom J Gastroenterol 2005;14:75-7. 